\title{
Structure and Property Correlations in FeS
}

\author{
S. J. Kuhn, ${ }^{1,2}$ M. K. Kidder, ${ }^{3}$ D. S. Parker, ${ }^{1}$ C. dela Cruz,${ }^{4}$ M. A. McGuire, ${ }^{1}$ W. M. Chance, ${ }^{1}$ Li Li, ${ }^{1}$ L. \\ Debeer-Schmitt, ${ }^{5}$ J. Ermentrout, ${ }^{1}$ K. C. Littrell, ${ }^{5}$ M. R. Eskildsen, ${ }^{2}$ A. S. Sefat ${ }^{*}$ \\ ${ }^{1}$ Materials Science \& Technology Division, Oak Ridge National Laboratory, Oak Ridge, TN 37831 \\ ${ }^{2}$ Department of Physics, University of Notre Dame, Notre Dame, IN 46556 \\ ${ }^{3}$ Chemical Sciences Division, Oak Ridge National Laboratory, Oak Ridge, TN 37831 \\ ${ }^{4}$ Quantum Condensed Matter Division, Oak Ridge National Laboratory, Oak Ridge, TN 37831 \\ ${ }^{5}$ Chemical and Engineering Materials Division, Oak Ridge National Laboratory, Oak Ridge, TN 37831
}

\begin{abstract}
For iron-sulfide (FeS), we investigate the correlation between the structural details, including its dimensionality and composition, with its magnetic and superconducting properties. We compare, theoretically and experimentally, the two-dimensional (2D) layered tetragonal (" $t$-FeS") phase with the 3D hexagonal (" $h$-FeS") phase. X-ray diffraction reveals iron-deficient chemical compositions of $t$ $\mathrm{Fe}_{0.93(1)} \mathrm{S}$ and $h$ - $\mathrm{Fe}_{0.84(1)} \mathrm{S}$ that show no low-temperature structural transitions. First-principles calculations reveal a high sensitivity of the $2 \mathrm{D}$ structure to the electronic and magnetic properties, predicting marginal antiferromagnetic instability for our compound (sulfur height of $z_{S}=0.252$ ) with an ordering energy of about $11 \mathrm{meV} / \mathrm{Fe}$, while the $3 \mathrm{D}$ phase is magnetically stable. Experimentally, $h$ - $\mathrm{Fe}_{0.84} \mathrm{~S}$ orders magnetically well above room temperature, while $t$ - $\mathrm{Fe}_{0.93} \mathrm{~S}$ shows coexistence of antiferromagnetism at $T_{N}$ $=116$ and filamentary superconductivity below $T_{c}=4 \mathrm{~K}$. Low temperature neutron diffraction data reveals antiferromagnetic commensurate ordering with wave vector $\boldsymbol{k}_{\boldsymbol{m}}=(0.25,0.25,0)$ and $0.46(2) \mu_{\mathrm{B}} / \mathrm{Fe}$. Additionally, neutron scattering measurements were used to find the particle size and iron vacancy arrangement of $t$-FeS and $h$-FeS. The structure of iron sulfide has a delicate relationship with the superconducting transition; while our sample with $a=3.6772(7) \AA$ is a filamentary superconductor coexisting with an antiferromagnetic phase, previously reported samples with $a>3.68 \AA$ are bulk superconductors with no magnetism, and those with $a \approx 3.674 \AA$ A show magnetic properties.
\end{abstract}




\section{Introduction}

In iron selenide, there is a close relationship between superconductivity and the chemical composition of the structure. This link between the properties and structure also exist for the other iron chalcogenides (hereafter $\mathrm{FeCh}$ ) of $\mathrm{FeS}$ and $\mathrm{FeTe}$, as they are difficult to synthesize stoichiometrically due to the existence of a wide solid-solution of phases close to the 1:1 ratio [1-3]. These binary tetragonal PbO-type iron chalcogenide structures (space group P4/nmm) are made of square-planar sheets of Fe, which are in a tetrahedral environment with the chalcogen atoms (Fig. 1a), with structural similarity to the superconducting iron arsenides. Small variations in Fe composition can generate great and wide ranging changes in the physical properties of FeCh. Shortly after the discovery of superconductivity in tetragonal 'FeSe' [4], a composition range of $\mathrm{Fe}_{1.01} \mathrm{Se}$ to $\mathrm{Fe}_{1.025} \mathrm{Se}$ was superconducting [5,6] with $\mathrm{Fe}_{1.01} \mathrm{Se}$ at $T_{c}=8 \mathrm{~K}$ and a structural transition (without magnetic order) at $T_{s}=90 \mathrm{~K} \mathrm{[7].} \mathrm{However,} \mathrm{slightly} \mathrm{more} \mathrm{iron-rich}$ $\mathrm{Fe}_{1.03} \mathrm{Se}$ is not a superconductor and does not undergo a structural transition [6]. The delicate relationship between structural details and magnetism is also prevalent in $\mathrm{FeTe}$. For $\mathrm{Fe}_{1.06} \mathrm{Te}$ to $\mathrm{Fe}_{1.17} \mathrm{Te}$ no superconductivity is found [8], but there is an ordered moment of $2 \mu_{\mathrm{B}}$ [9]. $\mathrm{Fe}_{1.141} \mathrm{Te}$ structurally changes from tetragonal to a mixed tetragonal/orthorhombic $(P m m n)$ phase below $76 \mathrm{~K}$, and it is orthorhombic below $T_{s}=56 \mathrm{~K}$ [8]; there is also incommensurate antiferromagnetic ordering below $T_{N}=63 \mathrm{~K}$ with a magnetic ordering wave-vector of $\boldsymbol{k}_{\boldsymbol{m}}=(0.38,0,0.25)$ [8]. However, $\mathrm{Fe}_{1.076} \mathrm{Te}$ is found to transform from tetragonal to a monoclinic $\left(P 2_{1} / \mathrm{m}\right)$ and antiferromagnetic phase below $T_{s}=T_{N}=75 \mathrm{~K}$ [8] with $\boldsymbol{k}_{\boldsymbol{m}}=(0.25,0,0.25)$ [8]. A slightly less Fe rich crystal structure with $\mathrm{Fe}_{1.068} \mathrm{Te}$ gives $T_{s}=T_{N}=67 \mathrm{~K}[10]$.

The tetragonal iron-sulfide phase ('mackinawite'; “t-FeS") also displays a sensitive interplay between structural details and properties. The recent finding of superconductivity $\left(T_{c}=4.5 \mathrm{~K}\right)$ in this phase [11] has created much excitement [12-18]. This material has been well studied in the field of geochemistry [1]. However, superconductivity was not found for many earlier studies of this tetragonal phase [19-23]. Due to the metastability of $t$-FeS, the use of the low-temperature hydrothermal method is important, for example by mixing $\mathrm{Fe}$ powder with $\mathrm{Na}_{2} \mathrm{~S}$ in an autoclave at $\sim 100{ }^{\circ} \mathrm{C}$ [11]. This method was further modified to yield single-crystals by leaching potassium from $\mathrm{K}_{0.8} \mathrm{Fe}_{1.6} \mathrm{~S}_{2}$ after heating in an autoclave with hexagonal binary powder $[13,15]$. Such recipes led to the superconducting ( $T_{c}=4$ to $4.5 \mathrm{~K}$ ) and metallic $\left(\rho_{300 \mathrm{~K}}=5 \mathrm{~m} \Omega \mathrm{cm}\right)$ samples $[11,13,15]$. The heat capacity results show Sommerfeld coefficient of $\gamma \approx 4$ to $5 \mathrm{~mJ} /\left(\mathrm{mol} \mathrm{K}^{2}\right)$ [15,17], with nodes in the superconducting gap and $2 d$-wave gaps [17]. Also, some muon spin relaxation $(\mu \mathrm{SR})$ measurements found small disordered magnetism below $20 \mathrm{~K}$ that co-exists with $T_{c}$ $=4.5 \mathrm{~K}$ [12]. Further $\mu \mathrm{SR}$ study shows a coexistent superconducting and magnetic phase in certain $t$-FeS samples [18]. Other methods of producing $t$-FeS included mixing powders of $\mathrm{FeCl}_{2}$ with $\mathrm{Na}_{2} \mathrm{~S}$ in basic solution and heating in an autoclave at $200{ }^{\circ} \mathrm{C}$ [20]; the resulting samples were ferromagnetic. Also, iron wire was mixed with $\mathrm{Na}_{2} \mathrm{~S}$ in an acidic solution and dried in an oxygen free environment or dropcast onto a gold plate [1,21,23], giving semiconducting behavior $\left(\rho_{300 \mathrm{~K}}=70 \mathrm{~m} \Omega \mathrm{cm}\right)$ with an iron-rich stoichiometry $\mathrm{Fe}_{1.27(1)} \mathrm{S}$ [21] and strong itinerant spin fluctuations [23]. Because of such wide physical properties in $\mathrm{FeS}$, the question of structural details and stoichiometry becomes important.

For our study, we compare the structure and properties of $t$-FeS with the hexagonal phase ('pyrrhotite', $P 6_{3} / \mathrm{mmc}$ ) that we denote as ' $h$-FeS' (Fig. 1b) The $t$-FeS system will easily phase transform into $h$-FeS under heating [19,22]. The crystal structure of the $h$-FeS is compared to the layered phase in Fig. 1: $h$-FeS contains triangular nets of $\mathrm{Fe}$, which are octahedrally coordinated with $\mathrm{S}$ giving a 3D structure [19,22]. Furthermore, $h$-FeS is a known antiferromagnet [24]. We report on the $t$-FeS phase, and compare its structural details and properties to those in the literature, and also to the $h$-FeS phase. The notion of structure-property relations in iron sulfide and the sensitivity to composition are investigated in this study for the first time. This article investigates $\mathrm{FeS}$ using temperature-dependent X-ray, neutron diffraction, and magnetization measurements, along with particle size, decomposition, and composition analyses. We draw conclusions from first principles calculations for $t$ - and $h$-FeS properties. 


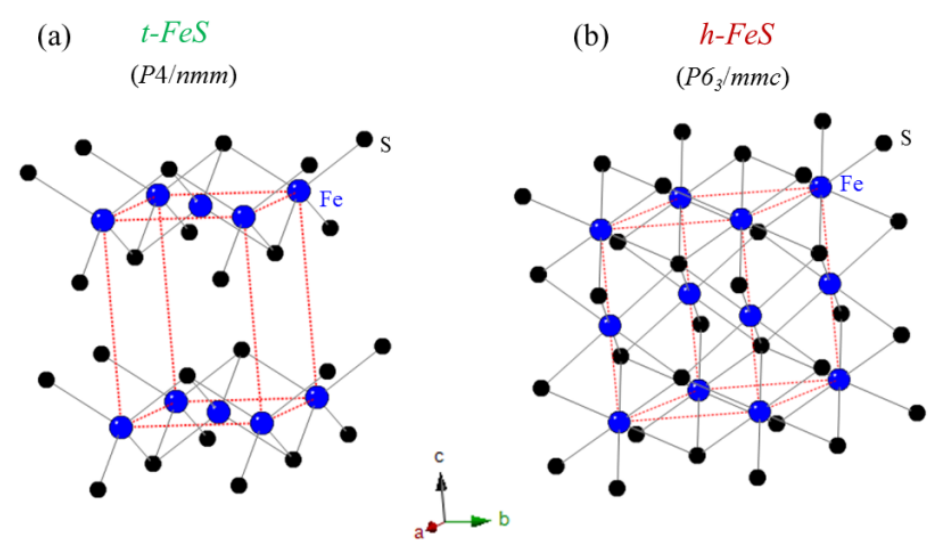

Fig 1. (Color online) Comparison of two crystal structures of FeS; unit cells are shown in red. (a) $t$-FeS has a layered crystal structure. (b) $h$-FeS is a 3D structure.

\section{Experimental}

Synthesis. $10 \mathrm{~g}$ of tetragonal $\mathrm{FeS}(t$-FeS) was prepared by a hydrothermal reaction, following the report of Lai [11]. 0.31 moles of $\mathrm{Na}_{2} \mathrm{~S} \cdot \mathrm{xH}_{2} \mathrm{O}$ (EM Science $\mathrm{x}=1.1$ ) were mixed with $55 \mathrm{~mL}$ DI water and 0.125 moles of Fe powder (Alfa 99.998\%). The reactants were sealed in a Teflon-lined $125 \mathrm{~mL}$ autoclave and baked at $100{ }^{\circ} \mathrm{C}$ for 6 days. After cooling naturally, the products were rinsed with DI water and acetone and dried in vacuum. Except for the drying, all steps were performed in air; this fitted $\mathrm{PbO}$ tetragonal P4/nmm structure. The hexagonal phase FeS $(h$-FeS) was prepared by standard solid state synthesis method. High purity powers of iron (Alfa 99.998\%) and sulfur (Alfa 99.9995\%) with stoichiometric ratio were mixed uniformly in glovebox and sealed into a silica tube, which was evacuated to a pressure of $10^{-2}$ Torr. The mixtures were heated up to $300{ }^{\circ} \mathrm{C}$, annealed for 12 hours, then slowly cooled to room temperature. The initially sintered sample was ground and pressed into round-shaped pellets $(10 \mathrm{~mm}$ diameter, $2 \mathrm{~mm}$ thick). The pellet was re-sealed in evacuated quartz tubes and sintered at $700{ }^{\circ} \mathrm{C}$ for 3 days and slowly cooled to room temperature. The obtained sample was black and stable in air.

Structure and composition. The images of the particles were taken using a Hitachi S3400 scanning electron microscope operating at $20 \mathrm{kV}$; the rough estimate of elemental compositions showed $\mathrm{Fe}: \mathrm{S}=1: 1$ using energy-dispersive X-ray spectroscopy (EDS). Powder X-ray diffraction measurements on both the tetragonal and hexagonal phases were conducted with a PANalytical X'Pert Pro MPD diffractometer using $\mathrm{Cu}-\mathrm{K}_{\alpha 1}$ radiation and an Oxford Phenix cryostat over a temperature range of $20 \mathrm{~K}$ to $300 \mathrm{~K}$. ICP analysis was performed on as-prepared samples of tetragonal $\mathrm{FeS}$ and hexagonal FeS. Samples were digested in high purity $2 \mathrm{M} \mathrm{HCl}$ (Alltrex) and subsequently diluted. A blank was tested using only $2 \mathrm{M}$ $\mathrm{HCl}$ to subtract any sodium present. During the digestion, $\mathrm{H}_{2} \mathrm{~S}$ was evolved and solid residue remained in the form of rhombic sulfur. The left over residue is likely the rhombic form of sulfur that occurs when digesting in a mineral acid. Sulfur composition was determined by Galbraith Labs, Knoxville, TN, using a Leco SC632 flame atomic absorption analyzer. This instrument combusts a small portion of sample in an oxygen atmosphere to create the combustion byproduct, $\mathrm{SO} ; \mathrm{SO}_{2}$ is then quantified by an infrared detector.

Electronic structure. First principles density functional theory calculations were performed using the linearized augmented plane wave code WIEN2K [25], within the Generalized Gradient Approximation [26]. Sphere radii of 1.85 Bohr (1 Bohr=0.529177 Angstrom) for $S$ and 2.26 for Fe were used and 
approximately $1000 \mathrm{k}$-points in the full Brillouin zone were used for the self-consistent calculations. An $\mathrm{RK}_{\max }$ (the product of the largest planewave expansion wavevector and the smallest sphere radius) of 7.0 was used. For the calculations of the densities-of-states, as many as 10,000 k-points were used.

Particle analysis and thermal-phase stability. Images of the powders were taken using a Hitachi S3400 Scanning Electron Microscope operating at $20 \mathrm{kV}$. For Small Angle and Ultra Small Angle Neutron Scattering (U/SANS) experiments, the FeS powder samples were loaded into dismountable quartz window cells with an adjustable path length and a diameter of $25 \mathrm{~mm}$. For the tetragonal structure sample, a path length of $0.21 \mathrm{~mm}$ was used and for the hexagonal, $1 \mathrm{~mm}$, which lead to multiple scattering impacting the accessible minimum $\mathrm{q}$ on the USANS. The measurements were done on beamline 1A USANS instrument at the Spallation Neutron Source (SNS) [27] and beamline CG-2, GPSANS at the High Flux Isotope Reactor (HFIR) at Oak Ridge National Laboratory (ORNL) [28]. The USANS data presented were measured using 3.6 ^ neutrons for horizontal angular collimation of 5 arcsec and 0.042 radians in the vertical direction. For the SANS measurements, data was collected at three detector settings $0.3,6$ and $18.5 \mathrm{~m}$ with a wavelength of $4.75 \AA$ with a $\Delta \lambda / \lambda=0.15$ covering a total $q$-range of $q \sim 0.0035 \AA^{-1}$ to $0.75 \AA^{-1}$. The beam diameter was $8 \mathrm{~mm}$ for both instruments to ensure that only the sample was being illuminated by the incoming neutrons. The SANS measurements were converted to an absolute scale by correcting for the calibrated attenuation applied to the empty direct beam. For scaling in the combined data, the USANS data were matched to appropriately slit smeared SANS data. With both USANS and SANS combined a large $q$-range of $5.3 \times 10^{-5}$ to $0.75 \AA^{-1}$ was used to probe the sample which allows the investigation of length scales from angstroms to micrometers. Thermogravimetric Analysis Mass Spectroscopy (TGA-MS) was acquired on a TA Instruments Q5000IR TGA and connected to Pfeiffer ThermoStar GSD 320 mass spectrometer via a heated capillary at $200{ }^{\circ} \mathrm{C}$. The samples (ca. $10-$ $20 \mathrm{mg}$ ) were loaded on pre-tared platinum high temperature pans, and purged in the TGA furnace for $2 \mathrm{~h}$ under nitrogen or argon then ramped from room temperature to either $350{ }^{\circ} \mathrm{C}$ or $1000{ }^{\circ} \mathrm{C}$ at $10^{\circ} \mathrm{C} / \mathrm{min}$. The mass spectrometer was set to scan masses $\mathrm{m} / \mathrm{z}=10-80$ at a scanning resolution of $50 \mathrm{~s}$.

Magnetism. Magnetization of the samples was performed in Quantum Design (QD) Magnetic Property Measurement System, on powders. Each sample was cooled to $2 \mathrm{~K}$ in zero-field, then the data were collected in field ( $20 \mathrm{G}$ or 1 Tesla) and warming to room temperature (zfc). Field-cooled data (fc) were collected in cooling for $t$-FeS. Neutron powder diffraction measurements were done on the HB2a High resolution powder diffractometer, housed at HFIR in ORNL. The measurements were done using a wavelength of $2.4127 \AA$ and a pre-monochromator, pre-sample and pre-detector collimation of open-21'12 ' respectively.

\section{Results and Discussions}

\section{i. Structure and composition}

The powder X-ray diffraction (XRD) data for $t$-FeS (Fig. 2a) is modeled using the PbO structure type $(P 4 / \mathrm{nmm})$ by Rietveld. The present sample contains a small Fe impurity of about 2 weight percent. We note that there is no measurement of $\mathrm{Fe}_{3} \mathrm{O}_{4}$. In the $t$-FeS phase, the variation in width of the reflections of different indices is notable. Reflections with no $c$-axis component, including (110), (200), and (220), are relatively sharp, with full width at half maximum of $0.17^{\circ}, 0.25^{\circ}$, and $0.31^{\circ}$, respectively. Reflections with both $a b$-plane and $c$-axis components are significantly broader. For example, the widths of the (101), (112), and (312) peaks are $0.36^{\circ}, 0.53^{\circ}$, and $0.71^{\circ}$, respectively. This is indicative of better crystallinity in $\mathrm{FeS}$ layers than between them, which may be a consequence of the low temperature synthesis requirements. XRD refinement gives $\mathrm{Fe}$ atomic site of $0.93(1)$ while $\mathrm{S}$ is fully occupied, giving $\mathrm{Fe}_{0.93(1)} \mathrm{S}$ phase composition for $t$-FeS (Table 1); we will denote the tetragonal phase $t$ - $\mathrm{Fe}_{0.93} \mathrm{~S}$ for the rest of the manuscript. We also checked the average composition of the entire sample using inductively coupled 
plasma (ICP) and combustion; the sample composition is (1.02)Fe:S:(0.32)Na. The most likely cause of sodium in the products is sodium hydroxide impurity on the surface of the powder due to imperfect washing of products, but it may also be sodium sulfide, sulfate, or carbonate. Other reports on $t$-FeS have shown varying stoichiometries. For a superconducting sample, (1.03)Fe:S was found using ICP atomic emission spectroscopy (ICP-AES) [11], while a non-superconducting sample gave (1.27)Fe:S in energydispersive X-ray spectroscopy (EDS) [21]. Independent of the iron deficiency, it may be crucial to keep the whole sample composition close to $\sim 1 \mathrm{Fe}: 1 \mathrm{~S}$ as to avoid impurity magnetic signals and transport grain boundaries that mask superconducting signals.

The refined room temperature lattice parameters of $t-\mathrm{Fe}_{0.93} \mathrm{~S}$ are $a=3.6779$ (8) $\AA$ and $c=5.0331(2) \AA$. Literature values on powders of superconducting $t$-FeS have longer $a$ lattice parameter compared to our data, while $c$ is comparable (Table 1). These superconducting $a$ values are reported as $3.6841 \AA$ [15], $3.6818 \AA$ [16], and $3.6802 \AA$ [11]. Literature values of non-superconducting $t$-FeS have shorter $a$ lattice parameters; they are 3.674 $\AA$ [20], $3.675 \AA$ [21], and 3.6735 $\AA$ [1]. Our $a=3.6779 \AA$ is in between these two sets of reported values and shows what appears to be filamentary superconductivity coexisting with antiferromagnetism (see below). The value of $a \geq 3.68 \AA$ may be crucial for bulk superconductivity, which is controlled by structural features such as iron stoichiometry and concentration of vacancies. As noted above, our $t$-FeS phase composition is $\mathrm{Fe}_{0.93(1)} \mathrm{S}$. The XRD data for $h$-FeS is shown in Fig. $\mathbf{2 b}$ and is modeled using the NiAs structure type $\left(\mathrm{P}_{3} / \mathrm{mmc}\right)$ by Rietveld. Small unindexed reflections are attributed to vacancy order, as is common for the hexagonal phase $[19,22,29]$. The vacancy ordering can be complicated, and is described in the literature by many hexagonal, monoclinic, and orthorhombic structures that vary depending on the iron stoichiometry. Collectively the phase is referred to as pyrrhotite and it includes $\mathrm{Fe}_{7} \mathrm{~S}_{8}$ stoichiometry. The structures can all be described by a $3+1$ superspace group where the modulation models Fe vacancy ordering; it is commensurate for certain vacancy concentrations [2931]. An appropriate vacancy order model could not be identified for the present sample. The atomic site refinement of $\mathrm{Fe}$ gives a value of $0.838(6)$ with $\mathrm{S}$ fully occupied, hence $\mathrm{Fe}_{0.84(1)} \mathrm{S}$ phase composition for $h$ FeS (Table 1), we will denote the hexagonal phase as $h-\mathrm{Fe}_{0.84} \mathrm{~S}$ for the rest of this manuscript. The ICPcombustion result on the entire sample is certainly evident of extra Fe-containing phases with (1.25)Fe:S. The lattice parameters at $300 \mathrm{~K}$ are $a=3.4436(1) \AA$ and $c=5.7262(2) \AA$. The temperature dependence of the lattice parameters for both $t-\mathrm{Fe}_{0.93} \mathrm{~S}$ and $h-\mathrm{Fe}_{0.84} \mathrm{~S}$ phases are shown in Fig. 3. No indication of a structural phase transition is seen down to $20 \mathrm{~K}$ for either sample. To illustrate this for $t$ - $\mathrm{Fe}_{0.93} \mathrm{~S}$, three reflections are compared at 20 and $300 \mathrm{~K}$ (Fig. 3a inset); no splitting or broadening is observed to occur between these two temperatures. The $a$ and $c$ lattice parameters decrease by 0.33 and $0.37 \%$, respectively, on cooling from 300 to $20 \mathrm{~K}$. For $h$-Fe $\mathrm{Fe}_{0.84} \mathrm{~S}$, the $a$ and $c$ lattice parameters (of the average structure) decrease by $0.5 \%$ and $0.15 \%$, respectively, over the same temperature range. In this material, there is an anomaly in the temperature dependence of $c$ near $75 \mathrm{~K}$, and we suspect that this may be related to an electronic/magnetic feature [32] rather than any change in vacancy order as there should be little atomic diffusion at that temperature. 

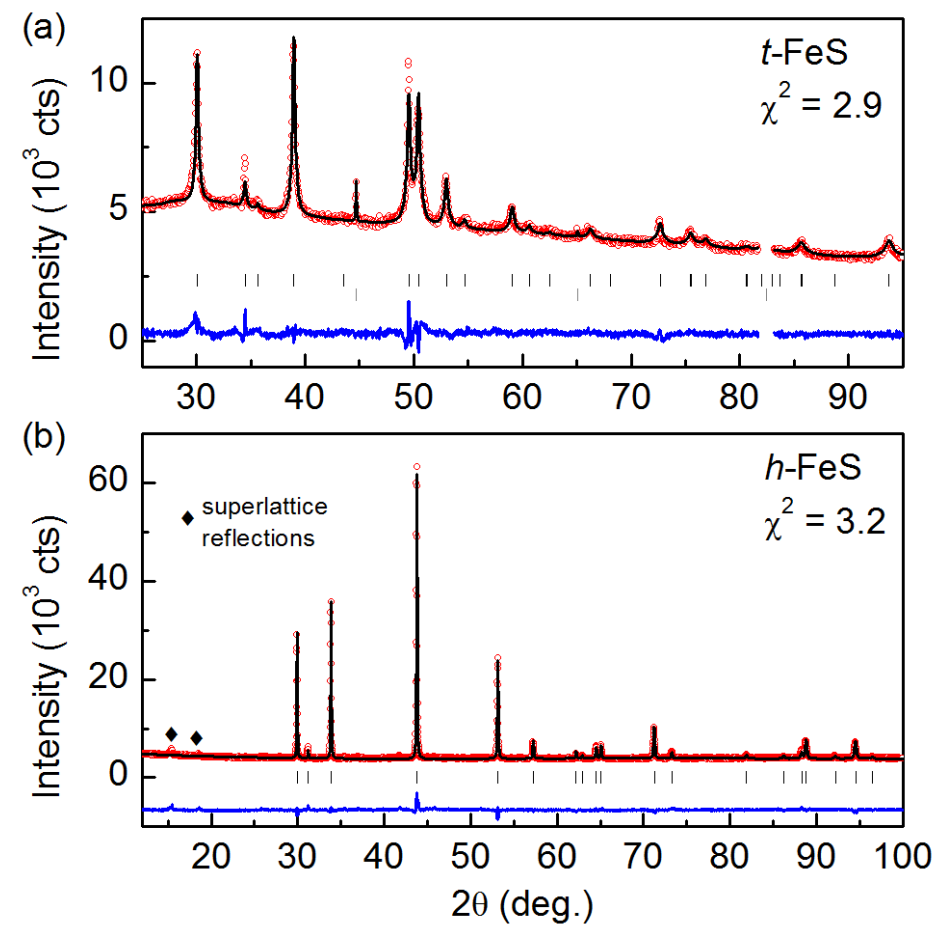

Fig.2. (Color online) Rietveld refinement results of room temperature powder X-ray diffraction patterns for (a) $t$-FeS and (b) $h$ FeS. In (a) the lower set of tics locate reflections from an $\mathrm{Fe}$ impurity; the reflection originating from the sample holder near $82^{\circ}$ is excluded. In (b) superlattice reflections arising from vacancy order are indicated; fits were performed only using the average structure (NiAs type) with vacancies randomly distributed.
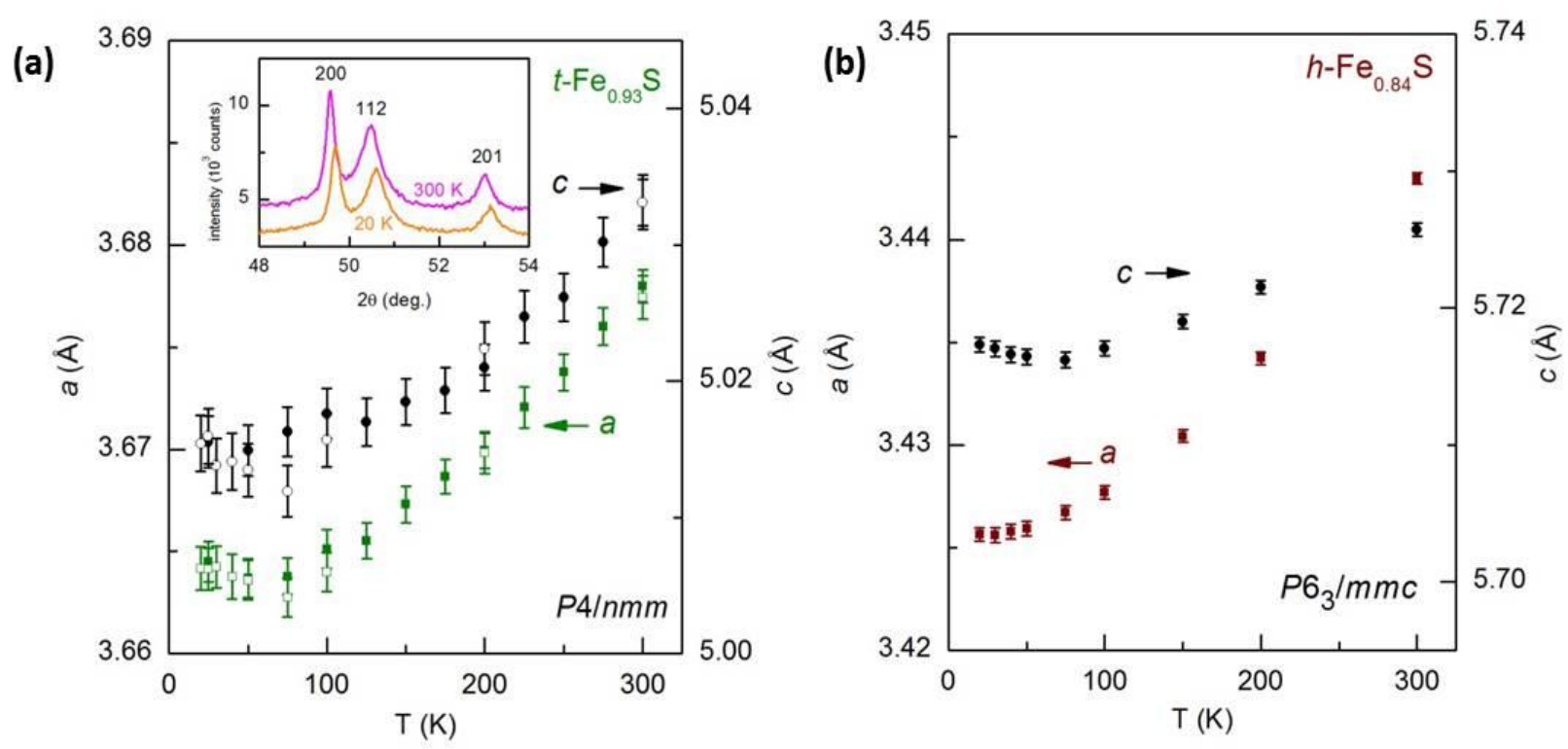

Fig. 3. (Color online) Temperature-dependent lattice parameters for $t$-FeS (a) and $h$-FeS (b). Symbols are measurements on different runs. 
Table 1. FeS powder phases, the lattice parameters, along with composition analyses, impurity phases, and possible superconducting transition temperature. The shaded rows are $h$-FeS. ${ }^{*}$ impurity signal may be from superlattice reflections from vacancy order in the main phase

\begin{tabular}{|c|c|c|c|c|c|}
\hline$a(\AA)$ & $c(\AA)$ & Fe:S, technique & Impurity & $T_{c}$ onset, zero (K) & Ref. \\
\hline- & - & 1:1, ICP-AES & - & 4.5 & 13 \\
\hline $3.6841(4)$ & $5.03440(9)$ & - & - & $4,2.4$ & 15 \\
\hline $3.6826(5)$ & $5.03440(9)$ & - & - & 3.9 & 15 \\
\hline $3.6818(1)$ & $5.0297(2)$ & - & - & 4.8 & 16 \\
\hline $3.6802(5)$ & $5.0307(7)$ & 1.03(2):1, ICP-AES & - & 5,4 & 11 \\
\hline $3.6772(7)$ & $5.032(1)$ & $\begin{array}{c}0.93(1): 1, \text { XRD } \\
\text { Fe:S:Na=1.02:1:0.32, ICP }\end{array}$ & $\begin{array}{l}\mathrm{Fe} \sim 2 \% \\
\quad \mathrm{Na}\end{array}$ & 4 & this study \\
\hline $3.674(3)$ & $5.0354(3)$ & - & $\mathrm{Fe}, \mathrm{FeS}_{2}$ & - & 20 \\
\hline $3.675(2)$ & $5.035(6)$ & 1.27(1):1, EDX & - & - & 21 \\
\hline $3.6735(4)$ & $5.033(7)$ & 0.99(1):1, ICP-AES & $\mathrm{Ti}, \mathrm{Mn}$ & - & 1 \\
\hline $3.447(2)$ & $5.747(2)$ & - & - & - & 31 \\
\hline $3.4437(2)$ & $5.7268(4)$ & $\begin{array}{c}0.84(1): 1, \text { XRD } \\
\text { Fe:S=1.25:1, ICP }\end{array}$ & $\begin{array}{c}\mathrm{Fe}_{\mathrm{x}} \mathrm{S}_{\mathrm{y}} \sim 2.6 \%{ }^{*} \\
-\end{array}$ & - & this study \\
\hline
\end{tabular}

\section{ii. $\quad$ Electronic structures}

We perform first principles calculations using the generalized gradient approximation on both $t-\mathrm{Fe}_{0.93} \mathrm{~S}$ and $h$ - $\mathrm{Fe}_{0.84} \mathrm{~S}$ structures. For our $t$ - $\mathrm{Fe}_{0.93} \mathrm{~S}$, X-ray diffraction refinement gives a sulfur height $\mathrm{z}_{\mathrm{S}}=0.252(3)$ at $20 \mathrm{~K}$, at which we find a marginal antiferromagnetic instability, with an ordering energy of just 11 $\mathrm{meV} / \mathrm{Fe}$ and staggered moment of $1.16 \mu_{\mathrm{B}}$. If $\mathrm{z}_{\mathrm{S}}$ is taken as 0.2602 , as found in the earlier experiment at $300 \mathrm{~K}$ [19], one finds a substantially larger ordering energy of $49 \mathrm{meV} / \mathrm{Fe}$ and staggered moment of 1.61 $\mu_{\mathrm{B}}$. Finally, if the structure is allowed to relax to an equilibrium position $\left(\mathrm{z}_{\mathrm{S}}=0.2356\right)$, one converges to a non-magnetic state. This FeS tetragonal structure is shown in Fig. 1a; the calculation assumed a "checkerboard" nearest-neighbor antiferromagnetic structure. The calculations indicate an extreme coupling of magnetism to structure, and that the tetragonal structure has a borderline nearest-neighbor antiferromagnetic instability, with the magnetism strongly dependent on the sulfur height [33]. This is in fact observed in the iron-based superconductors where the calculated magnetic properties are highly sensitive to the exact arsenic height [34]. In fact, the calculated magnetic properties are interrelated to stoichiometry and structure. The density-of-states in Fig. 4 (here we used our experimental structure) depict a rapid variation of $N(E)$ around the Fermi energy, and one might expect a dependence of magnetic character on stoichiometry. Accordingly, we have simulated further Fe-deficient $\mathrm{Fe}_{0.85} \mathrm{~S}$ (relaxed $\mathrm{z}_{\mathrm{S}}=0.2469$ ) within the virtual crystal approximation (VCA) and find an antiferromagnetic instability, with $41 \mathrm{meV} / \mathrm{Fe}$ ordering energy [35]. This calculation is done within the VCA-relaxed structure and in this 
case we find that relaxation strengthens the magnetism, rather than removing it, as in the stoichiometric case.

The $h$-FeS is much more magnetic; it is possible to stabilize several antiferromagnetic states along with a ferromagnetic state, all with energies several hundred meV per Fe below the non-magnetic state (Table 2). Hence this is much more of a local-moment system than the tetragonal phase, with much stronger magnetism. The reason for this divergence is strongly related to crystal structure, but for now we will focus on the calculated non-magnetic densities-of-states (we used our experimental structure with the sulfur height $z_{S}=0.252$; the hexagonal structure has no free coordinates). As is well known for the parent compounds of the Fe-based superconductors, in the tetragonal phase the Fermi level lies in the middle of a pseudogap, with Fermi-level DOS of 1.96/eV unit cell (both spins), and corresponding T-linear specific heat coefficient (c) of $2.31 \mathrm{~mJ} / \mathrm{mol}^{-\mathrm{K}^{2}}$. The DOS is dominated by Fe states, and assuming an Fe Stoner exchange parameter $I$ of $0.75 \mathrm{eV}$, with $2 \mathrm{Fe}$ per unit cell, the ferromagnetic Stoner criterion $I N\left(E_{F}\right)>1$ is not satisfied. This is consistent with the lack of a stable ferromagnetic solution and the marginal stability of the antiferromagnetic ground-state [36]. The situation is very different for the $h$-FeS, with $N\left(E_{F}\right)$ well over three times greater at 7.17/eV-u.c.; here there is no pseudogap and there is in fact a Van Hove singularity just $0.2 \mathrm{eV}$ below $E_{F}$. The Stoner parameter $I N\left(E_{F}\right)$ is 2.69 , indicating a strong tendency towards magnetism. This is consistent with the finding of numerous stable magnetic states, as depicted in Table 2. We find three separate antiferromagnetic states, along with a ferromagnetic state, to be stable relative to the non-magnetic state, with ordering energies of hundreds of meV per Fe. The great differences in electronic structure in the two phases can be directly tied to the crystal structure: while the tetragonal phase is well known to have 2D character, the hexagonal phase is much more 3D and yet also has strong one-dimensional character resulting from $c$-axis Fe-Fe coupling. The van Hove singularity results from this coupling and yields the higher $N\left(E_{F}\right)$, and hence stronger magnetic character in the hexagonal phase.
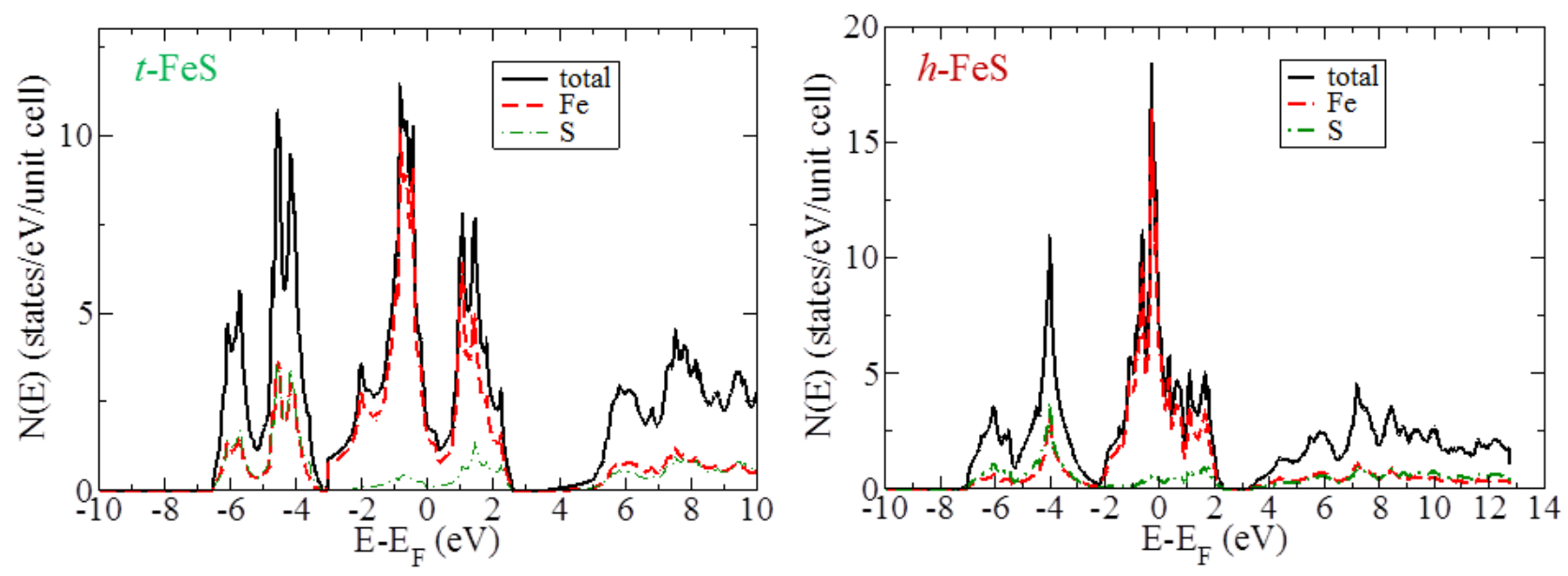

Fig. 4. (Color online) The calculated non-magnetic densities-of-states for the tetragonal (left) and the hexagonal phase (right) of FeS. 
Table 2: The calculated properties of $h$-FeS. FM refers to a ferromagnetic state, while AF1 refers to a state with nearest-neighbor Fe atoms (along the $c$-axis) antialigned and all next-nearest Fe neighbors (in the plane) ferromagnetically coupled. AF2 and AF3 have two and four, respectively, of these six nextnearest neighbors antiferromagnetically coupled.

\begin{tabular}{|c|c|c|}
\hline Ground-state & $\mathrm{E}-\mathrm{E}_{\mathrm{NM}}(\mathrm{meV} / \mathrm{Fe})$ & Moment $\left(\mu_{\mathrm{B}} / \mathrm{Fe}\right)$ \\
\hline $\mathrm{NM}$ & 0 & 0 \\
\hline $\mathrm{FM}$ & -338 & 2.65 \\
\hline $\mathrm{AF} 1$ & -475 & \pm 2.91 \\
\hline $\mathrm{AF} 2$ & -407 & \pm 2.60 \\
\hline $\mathrm{AF} 3$ & -428 & \pm 2.73 \\
\hline
\end{tabular}
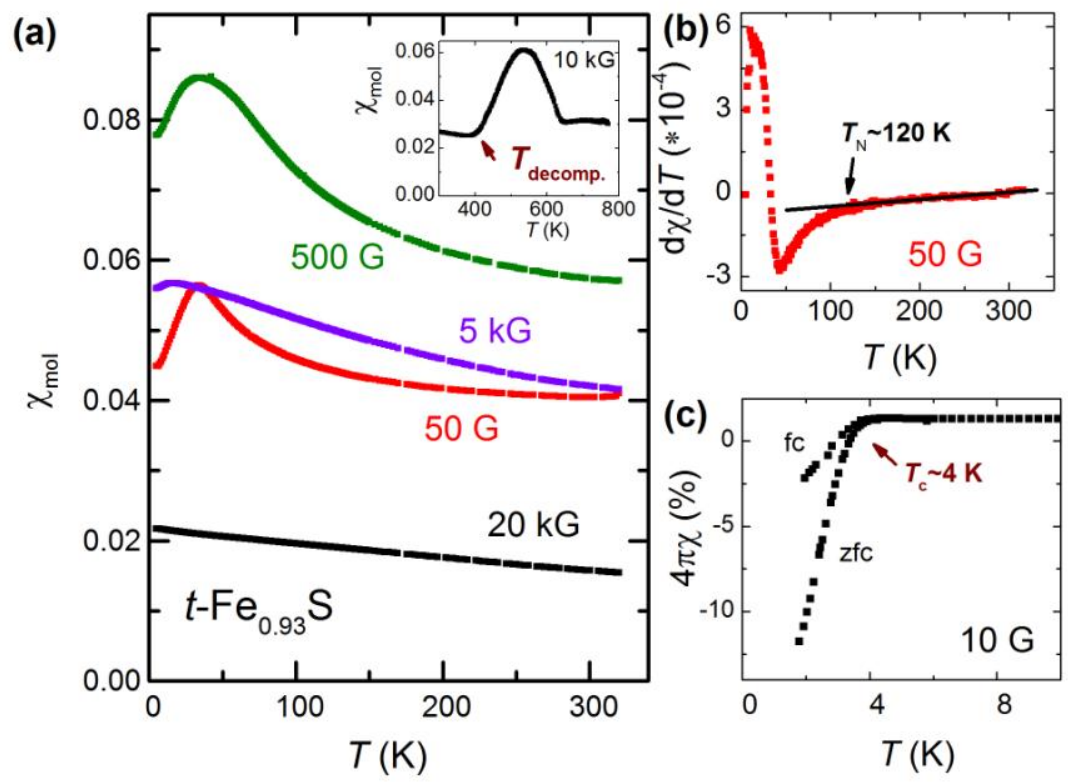

Fig. 5. (Color online) (a) Temperature dependence of magnetic susceptibility of $t$-Fe ${ }_{0.93} \mathrm{~S}$ under various applied fields. Inset shows the decomposition of the sample above $400 \mathrm{~K}$. (b) The $d \chi / d T$ curve at $50 \mathrm{G}$ shows a deviation from linear below $\sim 120 \mathrm{~K}$. (c) Diamagnetic behavior is observed below $4 \mathrm{~K}$ in zfc and fc data taken at $10 \mathrm{G}$.

\section{iii. Magnetism}


Temperature-dependent magnetic susceptibility $\chi$ results at several applied fields on $t-\mathrm{Fe}_{0.93} \mathrm{~S}$ show a broad feature starting below $\sim 120 \mathrm{~K}$ for $50 \mathrm{G}$ data (Fig. 5a, b), while diamagnetism appears below $4 \mathrm{~K}$ for $10 \mathrm{G}$ data (Fig. 5c). This sample is a filamentary superconductor for the small superconducting volume fraction of only $\sim 12 \%$ at $2 \mathrm{~K}$ (Fig. 5c). With higher field of $500 \mathrm{G}$, the peak is broadened and shifts to lower temperatures, and finally the low-temperature $\chi$ upturn at $\sim 15 \mathrm{~K}$ in $5000 \mathrm{G}$ data disappears in $20 \mathrm{kG}$ data (Fig. 5a). The inferred antiferromagnetic order may be quenched in powders with anisotropic magnetic behavior, similar to those that were seen in high-temperature antiferromagnets $\mathrm{BaMnPnF}(\mathrm{Pn}=\mathrm{As}, \mathrm{Sb}$ ) that even have moments of $3.65 \mu \mathrm{B}$ [37]. Higher temperature magnetization measurements on $t$ - $\mathrm{Fe}_{0.93} \mathrm{~S}$ (inset of Fig. 5a) gives a broad and large upturn starting just above room temperature, related to the thermal decomposition of this sample to the $h$-FeS phase with a greigite intermediary phase [15]. Literature data on $t$-FeS show Pauli paramagnetic behavior in the normal state below $50 \mathrm{~K}$ under low applied fields $\leq 10 \mathrm{G}[15,16]$. A report of magnetization on non-superconducting $t$-FeS featured a large and approximately temperature-independent behavior at $10 \mathrm{G}$, and a hysteresis in field, due to a ferromagnetic component without superconductivity [20]. Another non-superconducting sample also showed a ferromagnetic behavior with a drop in magnetic susceptibility below $\sim 30 \mathrm{~K}$, which was attributed to excess of iron incorporated in the structure [21].

Neutron diffraction data show a lone magnetic peak at $q=0.61 \AA^{-1}$ at $1.6 \mathrm{~K}$ that is absent for the high temperature pattern (inset of Fig. 6a). No other magnetic peaks are seen. The temperature-dependent order parameter measurement of the intensity of this peak is displayed up to $150 \mathrm{~K}\left(\right.$ Fig. 6a), with $T_{\mathrm{N}}=$ $116 \mathrm{~K}$. The magnetic peak position is consistent with a commensurate magnetic ordering wave vector of $\boldsymbol{k}_{\boldsymbol{m}}=(0.25,0.25,0)$. A magnetic structure model that best fits the data giving maximum intensity at the magnetic peak position is composed of spins having antiferromagnetic correlations within the $a b$ plane and ferromagnetic correlation along the $c$-axis. The width of the magnetic peak is resolution limited. This is the first evidence of long-range order in $t$-FeS, although it must be related to our structural details of $t$ -

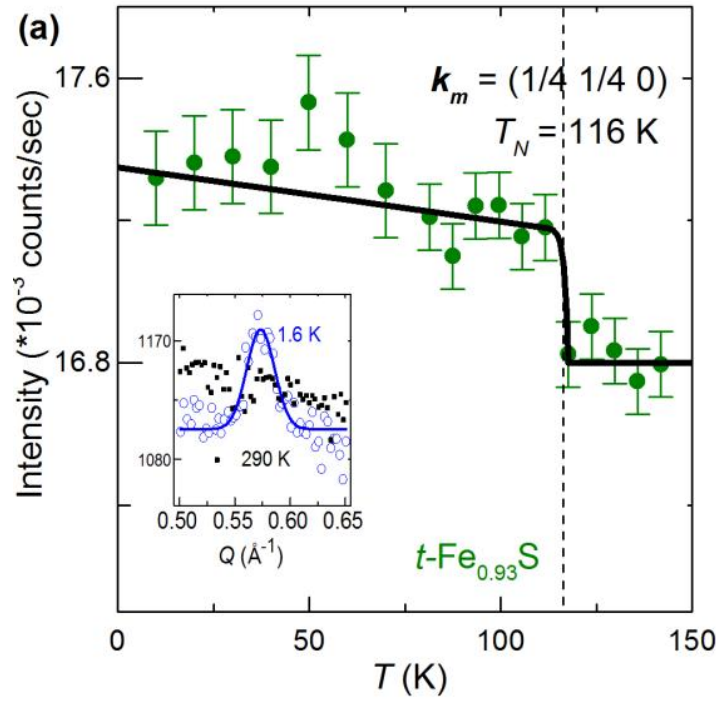

(b)

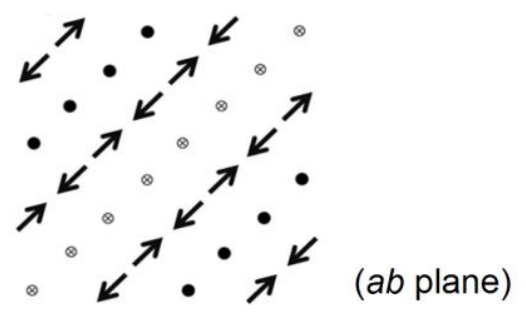

Fig. 6. (Color online) (a) Neutron powder diffraction of $t$ - $\mathrm{Fe}_{0.93} \mathrm{~S}$. The scattering intensity vs. temperature is plotted for $(0.25,0.25,0)$ wavevector, showing ordering below $116 \mathrm{~K}$; inset shows the scattering data below and above the $T_{\mathrm{N}}$. (b) Schematic of antiferromagnetic Fe sublattice with commensurate ordering; arrows portray the moment on the $\mathrm{Fe}$ site on $a b$-plane, and dots (crosses in gray) are the $\mathrm{Fe}$ moments parallel (antiparallel) to the $c$-axis. 
$\mathrm{Fe}_{0.93} \mathrm{~S}$. Fig. 6b shows the proposed magnetic structure of the Fe sublattice with arrows portraying the moment on the Fe site on $a b$-plane, and dots (crosses in gray) are the Fe moments parallel (antiparallel) to the $c$-axis. The spins form cycloids perpendicular to the $c$-axis with an ordered moment of $0.46(2) \mu_{\mathrm{B}} / \mathrm{Fe}$. Single crystal measurements are necessary to confirm this magnetic structure. For $h$-FeS in comparison, there are two susceptibility features: there is a spin transition in which the spins rotate along the $c$ axis at $450 \mathrm{~K}$ [24], and an accompanying structure transition to a different superstructure at $T_{N}=600 \mathrm{~K}[24,38]$.

\section{iv. Particle properties and thermal phase stability}

The iron vacancies and particle size of tetragonal and hexagonal FeS batches were investigated by measuring small and ultra-small angle neutron scattering (U/SANS) and looking at scanning electron microscope (SEM) images (Fig 7). The iron vacancies were compared between $t$-FeS and $h$-FeS by fitting the high- $q$ SANS data (Fig. 7a left inset). Small angle scattering uses the width and the shape of the forward diffraction peak to obtain information about particle, void and inclusion shapes and size distributions by comparison with model form factors. The data were compared with models for randomlydistributed roughly spherical objects, one-dimensional extended ( i.e. cylindrical) objects, and twodimensional (sheetlike) objects in a manner analogous to the estimation of crystal domain sizes from diffraction peak widths [39]. For $t$-FeS, a cylindrical fit was the best approximation of vacancies are ordered along a line [40] while for $h-\mathrm{FeS}$ using random oriented spheres approximation shows the data is best supported by random point defects. These iron vacancies could be related to differences in the lattice parameter size and could be directly responsible the filamentary superconductivity found in the t-FeS sample. This supports the idea of the sensitivity of $t$-FeS to its chemical properties [40]. Using U/SANS gave us the opportunity to investigate particles across a wide range of 0.1-1000 nm length scales; the combined data are plotted on a log-log scale (Fig. 7a). For both the tetragonal and hexagonal phases, there are variations in particle size and two general length scales are captured. The slope of the data and the positions of transition points between regions of different slopes describe the size and shape of the particles. For the tetragonal phase, there is a uniform particle size and shape described by low- $q$ slope of $\mathbf{I}$ $=-1.1(6)$, consistent with extended FeS sheets with thickness of $\sim 1400 \mathrm{~nm}$; the lateral extent of these sheets was too large to measure. At higher $q$, the slope of $\mathbf{I I}=-2.3$ corresponds to a fractal roughness on the surface of the particle. There is another transition point where the data go to III $=-4.5$ in slope, which could be due to diffuse interfaces, although interpretations of power-laws steeper than -4 is not clearly understood [41]. The right inset of Fig. 7a highlights an excess scattering over a power law of -4 on a linear scale. The data in the intermediate region is modeled as a distinct particle from which a particle size of $19 \mathrm{~nm}$ can be estimated, by the radius of gyration $\left(r_{g}\right)$ [42]. For the hexagonal phase, U/SANS reveals a superstructure that is too large to be measured, but the fits are consistent with rounder surfaces IV $=-3.1$, $\mathbf{V}=-5.5$. The excess scattering above a nominal power-law of 4, (Fig. 7a right inset) gives a particle size of $16 \mathrm{~nm}$. In the SEM image, roughly flat, plate-like particles with sides between 1000 and $5000 \mathrm{~nm}$ is supported for $t$-FeS (Fig. 7b), while $h$-FeS is larger and rounder, with the largest particle of approximately $10,000 \mathrm{~nm}$ in size. Smaller particle sizes were found with a number of techniques in literature. For example for the tetragonal phase, SEM and transmission electron microscopy (TEM) data show particle sizes between 250 and $50 \mathrm{~nm}$ [11,20,21,43], while XRD refinements and atomic force microscopy of the same samples reveal particle sizes between 42 and $4 \mathrm{~nm}[20,21,43]$.

Because the tetragonal and hexagonal iron sulfide structures are stabilized with different synthesis routes of hydrothermal vs. solid state reactions, and at different synthesis temperatures of $100{ }^{\circ} \mathrm{C}$ versus $700{ }^{\circ} \mathrm{C}$, respectively, we utilize the technique of thermogravimetric analysis-mass spectrometer (TGA-MS) here to monitor the difference between mass loss of these materials upon heating, and characterize the thermal decomposition products. A TGA report for $t$-FeS non-superconducting sample, synthesized in a solution similar to ref. [20], gave $\mathrm{SO}$ and $\mathrm{SO}_{2}$ species with a small $(<5 \%)$ weight change [42]; here we investigate 
a sample synthesized similar to the superconducting discovery paper [11]. Our as-prepared sample was stored in an argon-filled glove box after synthesis, and results are shown in Fig. 8. Very distinct decomposition behavior is observed below $350{ }^{\circ} \mathrm{C}$, with three mass losses up to this temperature. An initial mass loss to $150{ }^{\circ} \mathrm{C}$ was confirmed to be due to water $(2.9 \%)$ via mass spectrometry. In the temperature range of $150-350{ }^{\circ} \mathrm{C}$, the two losses are due to $\mathrm{H}_{2} \mathrm{O}, \mathrm{CO}_{2}$ and $\mathrm{SO}_{2}$ species, with $\mathrm{SO}_{2}$ being the major component. Deionized water was not purged during synthesis, leaving oxygen and carbon dioxide to be readily adsorbed onto the product or to react with $\mathrm{Na}_{2} \mathrm{~S}$ during the synthesis process. It is likely that sodium carbonate is also formed in the autoclave. Another sample of $t-\mathrm{Fe}_{0.93} \mathrm{~S}$ was 'dried' for 24 hours with a heat treatment under vacuum $\left(30 \mathrm{in}\right.$. $\mathrm{Hg}$ at $85^{\circ} \mathrm{C}$ ), and subsequent storage with $\mathrm{P}_{2} \mathrm{O}_{5}$ under argon. Heat treatment above $85{ }^{\circ} \mathrm{C}$ was observed to lead to decomposition in the sample, as seen by discoloration of the sample. This discoloration and crust formation at elevated temperatures supports the idea of a level of sodium, as was seen in ICP, in the form of sodium hydroxide and sodium carbonate impurity on the surface of the powder. Mass loss in the 'dried' sample is less than the as-prepared sample ( $4.601 \%$ vs. $7.090 \%$, respectively) up to the point of converging behavior $\left(\sim 350{ }^{\circ} \mathrm{C}\right)$. There is less water given off below $150{ }^{\circ} \mathrm{C}$, but a significant portion still remains (1.9\%). Compared to $t$ - $\mathrm{Fe}_{0.93} \mathrm{~S}$, the $h$ - $\mathrm{Fe}_{0.84} \mathrm{~S}$ sample shows less than $0.3 \%$ mass loss up to $350^{\circ} \mathrm{C}$, and less than $0.2 \%$ water loss.

(a)
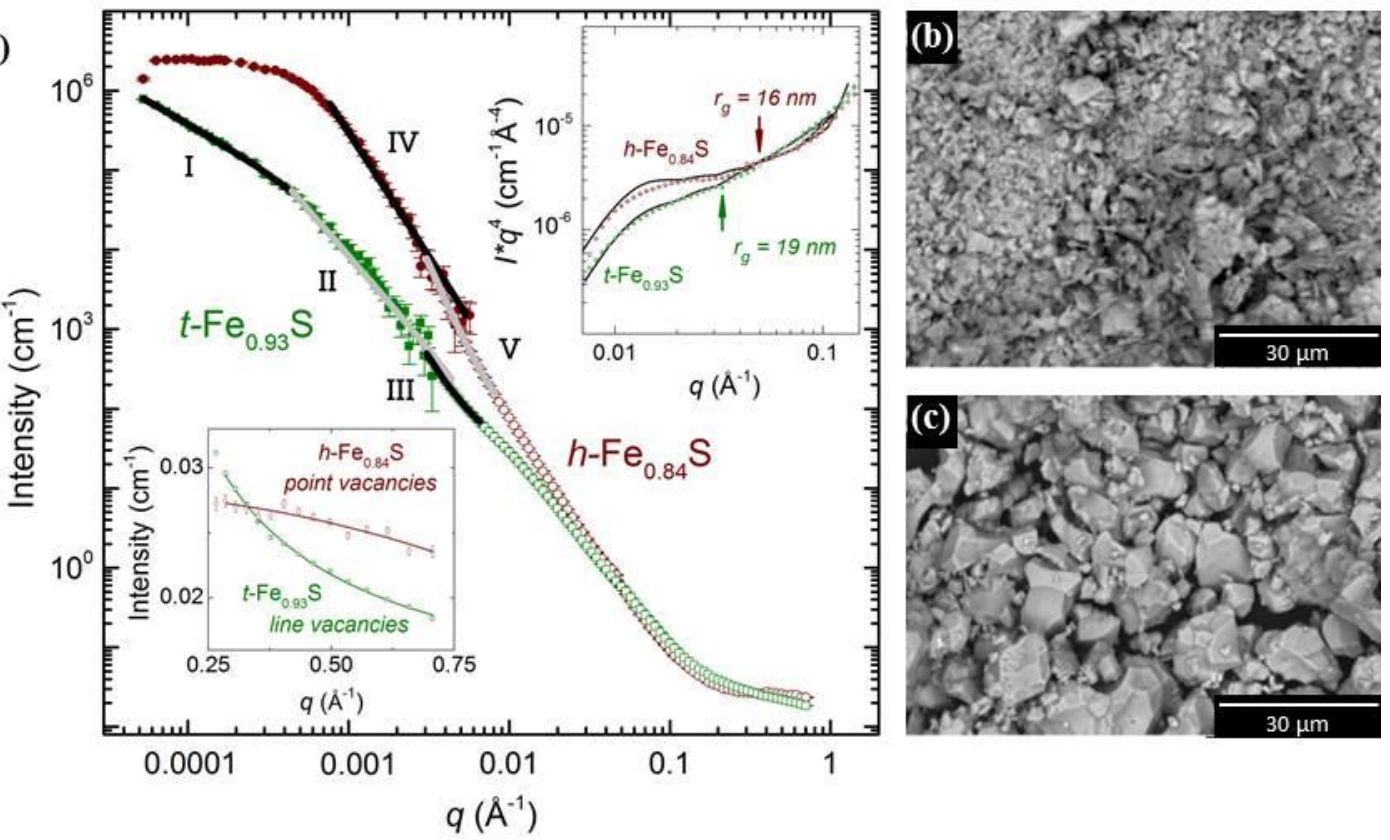

Fig. 7. (Color online) Particle sizes of the hexagonal and tetragonal phases are measured using USANS (filled symbols) and SANS (open symbols). Slopes displayed for the low- $q$ fits (I-V) describe the large-scale particle shapes, as described in the text. The high- $q$ data was fit using either randomly oriented spheres $(h-\mathrm{FeS})$ or cylinders $(t-\mathrm{FeS})$. The radius of gyration $\left(r_{g}\right)$ analysis reveals a similar particle size (right inset). Scanning electron microscope images of the powders are displayed for (b) $t$-FeS and (c) $h$-FeS. 


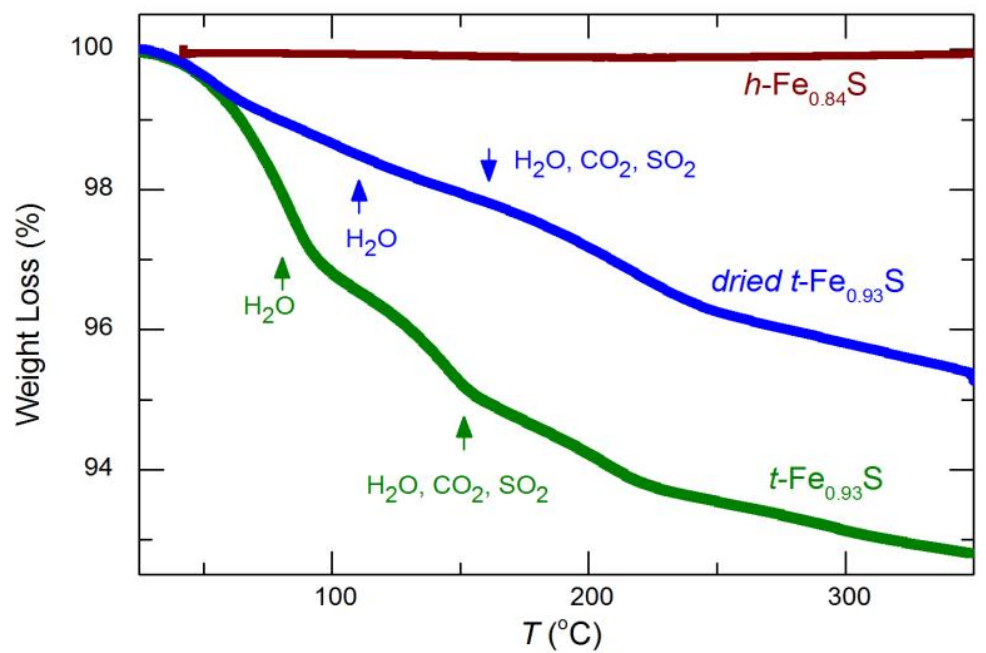

Fig. 8. Thermogravimetric analysis of $t$ $\mathrm{FeS}$, dried $t$-FeS, and $h$-FeS. Above 350 ${ }^{\circ} \mathrm{C}$ the samples show convergent behavior. Mass spectroscopy was used to identify the evaporating compounds at the temperatures indicated by arrows.

\section{Conclusion}

The substantial difference in magnetic and superconducting behavior of the different FeS phases here and in literature is directly tied to the structural details. Structurally, the $a$ lattice of $\geq 3.68 \AA$ may be a crucial for causing bulk superconductivity in $t$-FeS, which is relevant to iron stoichiometry and sulfur height from the iron plane. Although the $h$-FeS magnetically orders well above room temperature, our $t$ $\mathrm{FeS}$ is a filamentary superconductor below $T_{c}=4 \mathrm{~K}$, with a commensurate antiferromagnetic order below $T_{N}=116 \mathrm{~K}$. The calculations show that higher Fermi-level DOS in $h$-FeS gives robust magnetism, while the lower, and rapidly varying DOS in $t$-FeS leads to strongly sample-dependent superconducting and weak magnetic behavior. Line ordering of the iron vacancies was found for $t$-FeS. The $2 \mathrm{D}$ vs. 3D behavior of FeS is also supported by the particle morphology where we find particle sizes as small as $\sim 17$ $\mathrm{nm}$ for $t$-FeS and $h$-FeS that clump into 2D flakes or 3D boulders, respectively, at $\mu$ m length scales. TGA results show that unlike $h$ - $\mathrm{Fe}_{0.84} \mathrm{~S}, t$ - $\mathrm{Fe}_{0.93} \mathrm{~S}$ superconducting phase contains other species such as $\mathrm{H}_{2} \mathrm{O}$ and $\mathrm{CO}_{2}$. This study shows the important link between structures and properties in iron sulfides.

\section{Acknowledgement}

S.K. would like to acknowledge DOE Office of Science Graduate Student Research Program award for funding, which is administered by the Oak Ridge Institute for Science and Education for the Department of Energy under contract number DE-AC05-06OR23100. This work was primarily supported by the U.S. Department of Energy (DOE), Office of Science, Basic Energy Sciences (BES), Materials Science and Engineering Division (M.M, D.P. L.L, and A.S.) and Chemical Sciences, Geosciences, and Biosciences Division (M.K.K.). The work at ORNL's High Flux Isotope Reactor (HFIR) was sponsored by the Scientific User Facilities Division, Office of BES, U.S. DOE (C.D. and L.D.). This study was partially funded (W.C.) by ORNL's Lab-Directed Research \& Development of the Wigner Fellowship program. M.K. acknowledges Chemical Sciences, Geosciences, and Biosciences Division. J.E. would like to acknowledge Higher Education Research Experiences 'HERE' Program, as well as Ruth Ann Verell representing Allegheny College, for his summer-student opportunity at ORNL. MRE acknowledges support by the U.S. Department of Energy, Office of Basic Energy Sciences, under Awards No. DE-FG0210ER46783. 


\section{References}

1. A. R. Lennie, S. A. T. Redfern, P. F. Schofield, and D. J. Vaughan. Mineral. Mag. 59, 677-683 (1995).

2. H. J. Okamoto. Phase Equilib. 12, 383-389 (1991).

3. H. Okamoto and L. E. Tanner. Binary Alloy Phase Diag, Vol. 2 (eds Massalski, T. B. et al.) ASM International, 1990; pg. 1781-1783.

4. F. C. Hsu, J. Y. Luo, K. W. Yeh, T. K. Chen, T. W. Huang, P. M. Wu, Y. C. Lee, Y. L. Huang, Y. Y. Chu, D. C. Yan, and M. K. Wu. Proc. Natl. Acad. Sci. U.S.A. 105, 14262 (2008).

5. A. J. Williams, T. M. McQueen, and R. J. Cava. Sol. Sta. Comm. 149, 1507-1509 (2009).

6. T. M. McQueen, Q. Huang, V. Ksenofontov, C. Felser, Q. Xu, H. Zandbergen, Y. S. Hor, J. Allred, A. J. Williams, D. Qu, J. Checkelsky, N. P. Ong, and R. J. Cava. Phys. Rev. B 79, 014522 (2009).

7. T. M. McQueen, A. J. Williams, P. W. Stephens, J. Tao, Y. Zhu, V. Ksenofontov, F. Casper, C. Felser, and R. J. Cava. Phys. Rev. Lett. 103, 057002 (2009).

8. W. Bao, Y. Qiu, W. Huang, M. A. Green, P. Zajdel, M. R. Fitzsimmons, M. Zhernenkov, S. Chang, M. Fang, B. Qian, E. K. Vehstedt, J. Yang, H. M. Pham, L. Spinu, and Z. Q. Mao. Phys. Rev. Lett. 102, 247001.

9. M. D. Lumsden, and A.D. Christianson. J. Phys. Condens. Matter. 22, 203203 (2010).

10. S. Li, C. de la Cruz, Q. Huang, Y. Chen, J.W. Lynn, J. Hu, Y. L. Huang, F.C. Hsu, K. W. Yeh, M. L. Wu, and P. Dai. Phys. Rev. B 79, 054503 (2009).

11. X. Lai, H. Zhang, Y. Wang, X. Wang, X. Zhang, J. Lin, and F. Huang. J. Am. Chem. Soc. 137, 10148-10151 (2015).

12. S. Holenstein, U. Pachmayr, Z. Guguchia, S. Kamusella, R. Khasanov, A. Amato, C. Baines, H. H. Klauss, E. Morezoni, D. Johrendt, and H. Luetkens. Phys. Rev. B 93 140506(R) (2016).

13. H. Lin,Y. Li, Q. Deng, J. Xing, J. Liu, X. Zhu, H. Yang, and H. H.; Wen, Phys. Rev. B 93, 144505 (2016).

14. T. P. Ying, X. F. Lai, X. C. Hong, Y. Xu, L. P. He, J. Zhang, M. X. Wang, Y. J. Yu, F. Q.; Huang, and S. Y. Li. arXiv 1511, 07717 (2015).

15. C. K. H. Borg, X. Zhou, C. Eckberg, D.J. Campbell, S. R. Saha, J. Paglione, and E. E. Rodriguez. Phys. Rev. B 93, 094522 (2016).

16. U. Pachmayr, N. Fehn, and D. Johrendt. Chem. Comm. 52, 194-197 (2015).

17. J. Xing, H. Lin, Y. Li, S. Li, X. Zhu, H. Yang, and H. H. Wen. Phys. Rev. B 93, 104520 (2016).

18. F. K. K. Kirschner, F. Lang, C. V. Topping, P. J. Baker, F. L. Pratt, S. E. Wright, D. N. Woodruff, S. J. Clarke, and S. J. Blundell. ArXiv. 1607.00953 (2016)

19. A. R. Lennie, and D. J. Vaughan. Mineral Spec. 5, 117-130 (1996).

20. I. T. Sines, D. D. Vaughn, R. Misra, E. J. Popczun, and R. E. Schaak. Jou. Sol. Sta. Chem. 196, 17-20 (2012).

21. S. J. Denholme, S. Demura, H. Okazaki, H. Hara, K. Deguchi, M. Fujioka, T. Ozaki, T. Yamaguchi, H. Takeya, and Y. Takano. Mat. Chem. Phys. 147, 50-56 (2014).

22. A. R. Lennie, S. A. T. Redfern, P. E. Champness, C. P. Stoddart, P. F. Schofield, and D.J. Vaughan. Americ. Mineral 82, 302-309 (1997).

23. K. D. Kwon, K. Refson, S. Bone, R. Qiao, W. Yang, Z. Liu, and G. Sposito. Phys. Rev. B 83, 064402 (2011).

24. J. L. Horwood, M. G. Townsend, andA. H. Webster. Jou. Sol. Sta. Chem. 17, 35-42 (1976).

25. P. Blaha, K. Schwarz, G. Madsen, D. Kvasnicka, and J. Luitz. WIEN2k, An Augmented Plane Wave + Local Orbitals Program for Calculating Crystal Properties, Techn. Universität Wien, Austria, ISBN 3-9501031-1-2 (2001).

26. J. P. Perdew, K. Burke, M. Ernzerhof. Phys. Rev. Lett. 77, 3865 (1996).

27. J. M. Carpenter and M. Agamalian. J. Physics: Conf. Series 251, 12056 (2010).

28. G. D. Wignall, K. C. Littrell, W.T. Heller, Y. B. Melnichenko, K. M. Bailey, G. W. Lynn, D. A. Myles, V. S. Urban, M. V. Buchanan, D. L. Selby, and P. D. Butler. J. Appl. Cryst. 45, 990-998 (2012).

29. F. Li and H. F. Franzen. J. Alloys Comp. 238, 73-80 (1996).

30. D. M. Pasquariello, R. Kershaw, J. D. Passaretti, K. Dwight, and A. Wold. Inorg. Chem. 23, $872-874$ (1983).

31. Z. Izaola, S. Gonzalez, L. Elcoro, J. M. Perez-Mato, G. Madariaga, and A. Garcia. Acta. Crystall. B63, $693-702$ (2007).

32. M. Charilaou, J. Kind, D. Koulialias, P. G. Weidler, C. Mensing, J. F. Loffler, and A. U. Gehring. J. Appl. Phys. 118, 083903 (2015).

33. A. Subedi, L. Zhang, D. J. Singh, and M. H. Du. Phys. Rev. B. 78134514 (2008).

34. I. I. Mazin, M. D. Johannes, L. Boeri, K. Koepernik, and D. J. Singh. Phys. Rev. B 78, 085104 (2008).

35. A.R. Lennie; S. A. T. Redfern; P. F. Schofield; D. J. Vaughan. Mineralogical Magazine 59, 677 (1995).

36. D. Parker, M. G. Vavilov, A.V. Chubukov. and I. I. Mazin. Phys. Rev. B 80, 100508 (2009). 
37. B. Saparov, D. J. Singh, V. O. Garlea, and A. S. Sefat. Sci. Reports. 32154 (2013).

38. S. Sakkopoulos. J. Appl. Phys. 59, 3540 (1986).

39. B.T.M. Willis and C.J. Carlile. Experimental Neutron Scattering. "Small Angle Neutron Scattering." Oxford University Press , 2009.

40. G. E. Dieter. Mechanical Metallurgy. New York, NY, McGraw-Hill (1986).

41. P. W. Schmidt. J. Appl. Cryst. 24, 414-435 (1991).

42. P. B. Moore. J. Appl. Cryst. 13, 168-175 (1980).

43. H. Y. Jeong, J. H. Lee, and K. F. Hayes. Geochim. Cosmochim. Acta. 72, 493-505 (2008). 